\title{
WEIGHTED NORM INEQUALITIES AND UNIFORM ALGEBRAS
}

\author{
TAKAHIKO NAKAZI
}

(Communicated by Paul S. Muhly)

\begin{abstract}
Generalizations of the classical conjugation operator can be defined on a general uniform algebra. In this paper the $L^{2}$ weighted problems for the conjugation operators are considered and it is shown that the weights have forms similar to the classical case. The results in this paper have applications to concrete uniform algebras, for example, a polydisc algebra and a uniform algebra which consists of rational functions.
\end{abstract}

1. Introduction. Let $X$ be a compact Hausdorff space, let $C(X)$ be the algebra of complex-valued continuous functions on $X$, and let $A$ be a uniform algebra on $X$. Fix a nonzero complex homomorphism $\tau$ on $A$ and let $m$ be the representing measure for $\tau$ on $X$. Put $A_{0}=\{f \in A ; \tau(f)=0\}$ and $C_{0}=\{f \in C(X)$; $\left.\int_{X} f d m=0\right\}$. A uniformly closed subspace is called $A$-invariant if it invariant under multiplications by the functions in $A$. Suppose $I$ is a closed invariant subspace in $C_{0}$. For example, $A_{0}$ is such a subspace. It is clear that there exists a largest subspace $K_{0}$ contained in such a closed invariant subspaces, and, in fact, $K_{0}=\left\{f \in C_{0} ; \int_{X} f g d m=0\right.$ for all $\left.g \in A\right\}$.

The abstract Hardy space $H^{p}=H^{p}(m), 1 \leq p \leq \infty$, determined by $A$ is defined to be the closure of $A$ in $L^{p}=L^{p}(m)$ when $p$ is finite and to be the weak*-closure of $A$ in $L^{\infty}=L^{\infty}(m)$ when $p=\infty$. Let $v$ be a real function in $L^{\infty} ; \tilde{v}$ denotes the conjugate function, that is, $v+i \tilde{v}$ is in $H^{1}$ and $\int_{X} \tilde{v} d m=0$. Of course, it is possible that $v$ need not have the conjugate function $\tilde{v}$.

In this paper, we are interested in the nonnegative weight functions $w$ for which there is a positive constant $C$ such that

$$
\int_{X}|f|^{2} w d m \leq C \int_{X}|f+\bar{g}|^{2} w d m
$$

for all $f$ in $A$ and all $g$ in $I$. In the case $A$ is the disc algebra and $m$ is the normalized Lebesgue measure, such weight functions $w$ are well known when $I=A_{0}$ (cf. [4, 6]). It is difficult to give a characterization analogous to the disc algebra setting even if in the case of an annulus algebra. However, in previous papers $[\mathbf{7}, \S 3 ; \mathbf{8}, \S 4$; and $9, \S 7$ ], we determined weight functions $w$ when $w$ satisfies a stronger weighted norm inequality than (1), if $I=K_{0}$, for a general uniform algebra. That is,

(2) there exists a constant $\rho$ in $[0,1)$ and a function $k$ in $K_{0}^{\perp} \cap L^{1}$ such that $|w-k| \leq \rho w$.

When $A$ is the disc algebra, the Helson-Szegö theorem, which was proved in [1, Proposition 2], shows that $w$ satisfies (2) if and only if $w=e^{u+\tilde{v}}$, where $u$ and $v$ are

Received by the editors March 11, 1987.

1980 Mathematics Subject Classification (1985 Revision). Primary 46J15, 42B30, 30D55.

Research partially supported by Grant-in-Aid for Scientific Research, Ministry of Education. 
in $L^{\infty}$ and \|\|$_{\infty}<\pi / 2$. The main result in this paper shows this condition persists for a polydisc algebra and a uniform algebra which consists of rational functions.

2. Weighted norm inequalities. Let $P^{v}$ be the orthogonal projection from $L^{2}$ to $v H^{2}$ where $v$ is in $\mathscr{L}$, the set of all measurable positive functions on $X$ which are both bounded and bounded away from zero. Fix $I$ a closed invariant subspace in $C_{0} . \mathscr{P}_{I}^{v}$ denotes $P^{v}$ restricted to $v A+v^{-1} \bar{I}$. The following theorem is known in the case when $I=A_{0}$ or $I=K_{0}\left[9\right.$, Theorems 14 and $\left.14^{\prime}\right]$. For a general $I$ we can show a lifting theorem on $A \times \bar{I}$ as in the proof of [9, Theorem 2]. Then the following theorem follows as in the proof of [9, Theorem 14]. However, we will sketch of a direct proof, not using a lifting theorem. We need the following lemma which is probably known.

LEMMA 1. Let $M$ be a subspace of $L^{1}$. If for any $h$ in $M$

$$
\left|\int_{X} h w d m\right| \leq C \int_{X}|h| w d m
$$

then there exists a function $k$ in $M^{\perp} \cap L^{1}$ such that $|w-k| \leq C w$.

The proof is due to the Hahn-Banach theorem, that is, $\left(L^{1}\right)^{*}=L^{\infty}$. For the proof, $m$ be any probability measure.

THEOREM 1. Let $w$ be a nonnegative function in $L^{1}$. Then, $\mathscr{P}_{I}^{v}$ is uniformly bounded in $L^{2}(w)$ with respect to $v$ in $\mathscr{L}$ if and only if there exists a constant $\rho$ in $[0,1)$ and a function $k$ in $I^{\perp} \cap L^{1}$ such that $|w-k| \leq \rho w$.

PROOF. It is easy to see that $\left\|\mathscr{P}_{I}^{v}\right\| \leq C$ for all $v \in \mathscr{L}$ if and only if

$$
\left|\int_{X} f g w d m\right|^{2} \leq\left(1-C^{-1}\right) \int_{X}|v f|^{2} w d m \int_{X}\left|v^{-1} g\right|^{2} w d m
$$

for all $f \in A$, all $g \in I$, and all $v \in \mathscr{L}$. Hence the proof of the "if" part in the theorem is easy.

We shall prove the "only if" part. Let $h \in I$. Put $E_{n}=\{x \in X ; 0<|h(x)|<$ $1 / n\}, F_{0}=\{x \in X ; h(x)=0\}$, and $F_{n}=\{x \in X ;|h(x)| \geq 1 / n\}$. Define $v_{n}$ by

$$
v_{n}(x)= \begin{cases}1, & x \in E_{n}, \\ 1 / n, & x \in F_{0}, \\ |h(x)|^{1 / 2}, & x \in F_{n} .\end{cases}
$$

Then $v_{n} \in \mathscr{L}$ and

$$
\left|\int_{X} h w d m\right|^{2} \leq\left(1-C^{-1}\right) \int_{X}\left|v_{n}\right|^{2} w d m \int_{X}\left|v_{n}^{-1} h\right|^{2} w d m .
$$

Since $\lim _{n} \int_{X}\left|v_{n}\right|^{2} w d m=\int_{X}|h| w d m$ and $\lim _{n} \int_{X}\left|v_{n}^{-1}\right|^{2} w d m \leq \int_{X}|h| w d m$ for any $h \in I$,

$$
\left|\int_{X} h w d m\right| \leq\left(1-C^{-1}\right) \int_{X}|h| w d m .
$$

This proof is similar to that of the calculation of a norm of a Hankel operator in [7, Theorem 1]. Now the lemma above implies the "only if" part. 
Let $N_{\tau}$ denote the set of representing measures for $\tau$ on $X$. If $N_{\tau}$ is finite dimensional and $m$ is a core point of $N_{\tau}$, in Theorem 1 we can use $\mathscr{E}$ instead of $\mathscr{L}$, where $\mathscr{E}$ is a finite subgroup of $\mathscr{L}$ (cf. [7, Proposition 5, 9, Theorem 14]). Moreover, if $m$ is a unique logomodular measure we can show that if $\mathscr{P}_{I}^{v}$ is bounded for each $v$ in $\mathscr{E}$, then $\mathscr{P}_{I}^{v}$ is uniformly bounded with respect to $\mathscr{E}$ (cf. [7, Theorem $6 ; 9$, Theorem 15]). If $A$ is the disc algebra and $m$ is the normalized Lebesgue measure, then $\mathscr{E}$ consists of nonzero positive constants and hence the Helson-Szegö theorem follows if $I=A_{0}$.

It is interesting to point out that Theorem 1 is completely general. However, we wish to find the weight functions $w$ for which $\mathscr{P}_{I}=\mathscr{P}_{I}^{1}$ is bounded (only when $v=1)$. For this purpose we introduce a constant $\gamma_{0}$ which was first used in $[8$, $\S 1$. Denoting the coset of an $f$ in $\left(L^{\infty}\right)^{-1}$ in $\left(L^{\infty}\right)^{-1} /\left(H^{\infty}\right)^{-1}$ by $(f)$, define $\|(f)\|=\inf \left\{\|g\|_{\infty}\left\|g^{-1}\right\|_{\infty} ; g \in(f)\right\}$ and $\gamma_{0}=\sup \left\{\|(f)\| ;(f) \in\left(L^{\infty}\right)^{-1} /\left(H^{\infty}\right)^{-1}\right\}$. If $A$ is the disc algebra then $\gamma_{0}=1$ and if $H^{\infty}$ is the algebra of bounded analytic functions on a multiply connected domain, then $\gamma_{0}$ is finite $[8, \S 2]$. The following theorem is known in the case of $I=A_{0}$ or $I=K_{0}$ (see $[8$, Corollary $1 ; \mathbf{9}$, Theorem 16]). The proof is almost the same if we use Theorem 1 .

THEOREM 2. Let $w$ be a nonnegative function in $L^{1}$. If $\mathscr{P}_{I}$ is bounded in $L^{2}(w)$ and if $\left\|\mathscr{P}_{I}\right\|=C$, then there exists a function $k$ in $I^{\perp} \cap L^{1}$ such that $|w-k| \leq \rho w$ and $\rho=\gamma_{0} \sqrt{C^{2}-1} / C$. Hence if $C<\gamma_{0} / \sqrt{\gamma_{0}^{2}-1}$, then $\rho<1$.

3. Characterizations of weights. By Theorems 1 and 2 we are interested in the weights $w$ which satisfy (2). $K_{0}^{\perp} \cap L^{1}$ coincides usually with $H^{1}$ and then (2) is the following:

(I) There exists a constant $\rho$ in $[0,1)$ and a function $k$ in $H^{1}$ such that $|w-k| \leq$ $\rho w$.

We will consider the equivalence of (I) and the following:

(II) $w=e^{u+\tilde{v}}$, where $u$ and $v$ are in $L^{\infty}$ and $\|v\|_{\infty}<\pi / 2$.

For many examples, (I) is equivalent to (II) (see §4).

LEMMA 2. Let $w$ be a nonnegative function and $k$ a function in $L^{1}$. For a constant $\rho$ in $[0,1),|w-k| \leq \rho w$ if and only if $w=e^{u}|k|$, where $|\arg k(x)| \leq \pi / 2-\varepsilon$ if $k(x) \neq 0$ and $\varepsilon>0$, and $u$ is in $L^{\infty}$.

ProOF. (see [1, Proposition 2] for the disc algebra). Suppose $|w-k| \leq \rho w$ and put

$$
\eta(x)= \begin{cases}(w(x)-k(x)) / \rho w(x) & \text { if } w(x)>0 \\ 1 & \text { if } w(x)=0\end{cases}
$$

then $|\eta(x)| \leq 1, k(x)=\rho w(x)(1 / \rho-\eta(x))$ if $w(x)>0$, and $k(x)=0$ if $w(x)=0$. Since $1 / \rho>1, \operatorname{Re} k(x) \geq 0$ and $|\arg k(x)| \leq \pi / 2-\varepsilon$ for some $\varepsilon>0$ if $k(x) \neq 0$. Put

$$
u(x)= \begin{cases}-\log \rho|1 / \rho-\eta(x)| & \text { if } w(x)>0, \\ 0 & \text { if } w(x)=0\end{cases}
$$

then $w=e^{u}|k|$, and this implies the part of "only if." Conversely suppose $w=$ $e^{u}|k|$ and $|\arg k| \leq \pi / 2-\varepsilon$. We may assume $e^{u} \geq 1$. Hence if $w(x)>0$, then $\left|1-e^{-u} k(x) /\right| k(x)|| \leq \rho_{2}<1$ for some $\rho_{2} \in[0,1)$ because $|1-k(x) /| k(x)|| \leq \rho_{1}<1$. Thus the "if" part follows. 
Let $L=L(m)$ be the set of measurable functions $f$, such that $\log ^{+}|f|$ is integrable. We define a metric in $L$ by setting

$$
\begin{aligned}
d(f, g)= & \inf _{t>0}[t+m(\{x \in X ;|f(x)-g(x)| \geq t\})] \\
& +\int_{X}\left|\log ^{+}\right| f\left|-\log ^{+}\right| g|| d m .
\end{aligned}
$$

With the metric $d, L$ becomes a complete metric space. The Hardy algebra $H=$ $H(m)$ is the closure of $A$ in $L(m)$. $H$ contains each of $H^{p}, 0<p \leq \infty$. In many examples (see $\S 4$ ), $H \cap L^{p}=H^{p}$ and the following is valid:

(3) If $f$ is a function in $H^{1}$ such that $\operatorname{Re} f \geq 0$, then $1 / f$ belongs to $H$.

LEMMA 3. Suppose $H^{1}$ has the property (3). If $f$ is a function in $H^{1}$ and $\operatorname{Re} f \geq 0$, then $\log f$ belongs to $H \cap L^{1}$, where $\log z$ is the principal branch of the logarithm.

ProOF. (see [2, Theorem 12 and 10, Proposition 5] for $N_{\tau}=\{m\}$ ). By property (3), $1 /(f+\varepsilon) \in H \cap L^{\infty}$ and $\operatorname{Re}(1 / f+\varepsilon) \geq 0$ for any $\varepsilon>0$. Using a Taylor series expansion,

$$
\log (1 / f+\varepsilon)=\log |1 / f+\varepsilon|+i \arg (1 / f+\varepsilon)
$$

belongs to $H \cap L^{\infty}$. Now, for every $x, \log (1 / f(x)+\varepsilon) \rightarrow \log (1 / f(x))$ as $\varepsilon \rightarrow 0$. Further $\mid \arg (1 / f(x)+\varepsilon \mid \leq \pi / 2$ and as $\varepsilon \rightarrow 0, \log |1 / f(x)+\varepsilon| \rightarrow \log |1 / f(x)|$. $\log |1 / f(x)+\varepsilon| \in L^{1}$ and, by property (3), $\log |1 / f(x)| \in L^{1}$. Given $\varepsilon_{0}>0$ for $0<\varepsilon<\varepsilon_{0}$, we have

$$
|\log | 1 / f(x)+\varepsilon|\leq| \log |1 / f(x)||+| \log \left|1 / f(x)+\varepsilon_{0}\right| \mid .
$$

Applying Lebesgue's dominated convergence theorem we see that $\log (1 / f+\varepsilon) \rightarrow$ $\log (1 / f)$ in $L^{1}$ as $\varepsilon \rightarrow 0$ and hence $\log (1 / f)$ is in $H \cap L^{1}$.

THEOREM 3. Suppose $H \cap L^{1}=H^{1}$. Let $w$ be a nonnegative function in $L^{1}$; then the following are valid.

(i) If $w$ satisfies (II), then $w$ satisfies (I).

(II) Suppose $H^{1}$ has the property (3). If $w$ satisfies (I), then $w$ satisfies (II).

PROOF. (i) Since $v$ in (II) is in $L^{\infty}, \tilde{v}-i v \in H^{1}$, and there exist $\tilde{v}_{n}-i v_{n} \in A$ such that $v_{n} \rightarrow v$ in $L^{1}$ and $\tilde{v}_{n} \rightarrow \tilde{v}_{n}$ a.e. By Lemma 2.4 in [3, p. 123], $e^{\tilde{v}-i v} \in H \cap L^{1}$. Set $k=e^{\tilde{v}-i v}$; then by the hypothesis $k \in H^{1}, w=e^{u}|k|$ and $|\arg k| \leq \pi / 2-\varepsilon$ for some $\varepsilon>0$. Now Lemma 2 implies (I).

(ii) By Lemma 2, $w=e^{u}|k|$, where $|\arg k(x)| \leq \pi / 2-\varepsilon$ if $k(x) \neq 0$ and $\varepsilon>0$, and $u$ is in $L^{\infty}$. By Lemma $3, \log k=\tilde{v}-i v \in H^{1}, v(x)=\arg k(x)$ and so $|k|=e^{\tilde{v}}$. This implies (II).

4. The finite-dimensional case. Suppose $N_{\tau}$ is finite dimensional and $m$ is a core point of $N_{\tau}$. Let $N^{\infty}$ be the real annihilator of $A$ in $L_{R}^{\infty}$ and $N_{c}^{\infty}=N^{\infty}+i N^{\infty}$ (cf. [3, p. 109]). Suppose $N^{\infty} \subset C(X)$. Then $K_{0}=A_{0}+N_{c}^{\infty}$ and hence $K_{0}^{\perp} \cap L^{1}=$ $H^{1}$ by $[3$, p. 106].

THEOREM 4. Let $w$ be a nonnegative function in $L^{1}$. Then $\mathscr{P}_{I}^{v}$ is uniformly bounded in $L^{2}(w)$ with respect to $v$ in $\mathscr{E}=\exp N^{\infty}$ if and only if $w=e^{u+\tilde{v}}$, where $u$ and $v$ are in $L^{\infty}$ and $\|v\|_{\infty}<\pi / 2$.

Proof. By [3, V, Theorem 4.2], $H \cap L^{1}=H^{1}$. By the argument of A. Devinatz in [2] $H^{1}$ has property (3). For if $f \in H^{1}$ and $\operatorname{Re} f \geq 0$, then, by [3, V, Lemma 
2.4], $e^{t f} \in H \cap L^{\infty}=H^{\infty}$ for $t \leq 0$. By the proof of [2, Lemma 4], $1 /(f+\varepsilon) \in H^{\infty}$ for any $\varepsilon>0$. By the proof of [2, Theorem 12], we can show that $\log |f| \in L^{1}$ and

$$
-\infty<\log \left|\int f d m\right|=\sup _{\mu \in S} \int \log |f| d \mu,
$$

where $S$ is the set of logmodular measures in $N_{\tau}$. By [3, V, Theorem 5.2], $f^{-1}$ belongs to $H$. By Theorem 3, (I) and (II) are equivalent. Now Theorem 2 and the remark above implies the theorem.

If $\operatorname{dim} N_{\tau}=0$, Theorem 4 is due to Y. Ohno [10], A. Devinatz [2], and I. I. Hirschman, Jr. and R. Rochberg [5].

5. Concrete examples in which (I) and (II) are equivalent. In the disc algebra, $K_{0}^{\perp} \cap L^{1}=H^{1}$, (I) and (II) are equivalent. We shall give examples in which these are valid.

(i) Let $Y$ be a compact subset of the plane whose complement has a finite number of components and let $R(Y)$ be the uniform closure of the rational functions in $C(Y)$. Let $A$ be the restriction of $R(Y)$ to its Shilov boundary $X$; then $M_{A}=Y$. If $\tau \in M_{A}$ is in the interior of $Y$ (we assume it is nonempty) and $m$ is harmonic measure, then $m$ is a unique logmodular measure of $N_{\tau}$ and $\operatorname{dim} N_{\tau}=n<\infty[3$, p. 116]. Then $N^{\infty} \subset C(X)$. Theorem 4 applies to this situation.

(ii) Let $A$ be a subalgebra of the disc algebra which contains the constants and which has finite codimension. If $\tau(f)=f(0)$ for $f$ in $A$ and $m$ is the normalized Lebesgue measure on the unit circle $T$, then $N_{\tau}$ is finite dimensional, $m$ is a core point of $N_{\tau}, N^{\infty} \subset C(X)$. Theorem 4 applies to this situation.

(iii) The unit polydisc $U^{n}$ and the torus $T^{n}$ are cartesian products of $n$ copies of the unit disc $U$ and of the unit circle $T$, respectively. $A\left(U^{n}\right)$ is the class of all continuous complex functions on the closure $\bar{U}^{n}$ of $U^{n}$ with holomorphic restriction to $U^{n}$ is holomorphic there. Let $A=A\left(U^{n}\right) \mid X$ and $X=T^{n}$. This is the so-called polydisc algebra. For simplicity we assume $n=2$. Let $m$ be the normalized Lebesgue measure; then $m$ is a representing measure for $\tau$ on $T^{2}$ where $\tau(f)=f(0)$ and $0 \in U^{2}$. Then $K_{0}=\left\{f \in C\left(T^{2}\right) ; \hat{f}(n, m)=0\right.$ for $(-n,-m)$ in $\left.Z_{+}^{2}\right\}$ where $Z_{+}^{2}$ denotes the set of all pairs $(n, m)$ of nonnegative integers $n$ and $m$. $K_{0}^{\perp} \cap L^{1}=H^{1}$ and $H^{1}$ has property (3). For if $f$ is a function in $H^{1}$ such that $\operatorname{Re} f \geq 0$, then by [11, Theorems 4.4.6 and 4.4.9], $f$ is outer; that is, $\log |f(0)|=\int_{T^{2}} \log |f| d m$. Then $1 / f$ is analytic on $U^{n}$ and $1 / f$ belongs to $H$ (see [11, Theorems 3.3.5 and 3.3.6]).

I am very grateful to the referee who improved the exposition in the first draft of this paper.

\section{REFERENCES}

1. R. Arocena, M. Cotlar and C. Sadosky, Weighted inequalities in $L^{2}$ and lifting properties, Academic Press, New York, 1981, pp. 95-128.

2. A. Devinatz, Conjugate function theorems for Dirichlet algebras, Rev. Un. Mat. Argentina 23 (1966), 3-30.

3. T. W. Gamelin, Uniform algebras, Prentice-Hall, Englewood Cliffs, N.J., 1969.

4. H. Helson and G. Szegö, A problem in prediction theory, Ann. Mat. Pura Appl. 51 (1960), 107-138.

5. I. I. Hirschman, Jr. and R. Rochberg, Conjugate function theory in weak-* Dirichlet algebras, J. Funct. Anal. 16 (1974), 359-371. 
6. R. A. Hunt, B. Muckenhoupt and R. L. Wheeden, Weighted norm inequalities for the conjugate function and Hilbert transforms, Trans. Amer. Math. Soc. 176 (1973), 227-251.

7. T. Nakazi, Norms of Hankel operators and uniform algebras, Trans. Amer. Math. Soc. 299 (1987), 573-580.

8. __ Norms of Hankel operators and uniform algebras. II, Tôhoku Math. J. 39 (1987), 543-555.

9. T. Nakazi and T. Yamamoto, $A$ lifting theorem and uniform algebras, Trans. Amer. Math. Soc. 305 (1988), 79-94.

10. Y. Ohno, Remarks on Helson-Szegö problems, Tôhoku Math. J. 18 (1965), 54-59.

11. W. Rudin, Function theory in polydiscs, Benjamin, New York, 1969.

Department of Mathematics, FaCulty of SCIEnCE, HokKaido University, SAPPORO 060, JAPAN 